Reprod. Nutr. Dévelop., 1988, 28 Suppl. n¹, 155-156

\title{
Variations qualitatives et quantitatives des acides biliaires dans la bille vésiculaire du lapin en fonction de la teneur en parois cellulaires du régime
}

\author{
A. HOMRANI, Germaine DEMAUX, Louise GUEMON, F. GALLOUIN
}

Station de Nutrition et d'Alimentation, I.N.A.-P.G., 16. rue Claude-Bernard, 75237 Paris Cedex 05

Summary. Two experiments were carried out to study the biliary acids in the rabbit gall bladder. The first studied the effect of diet : commercial rabbit feed, $70 \%$ lucerne diet, $70 \%$ beet pulp diet. The second experiment studied biliary acids two groups of young rabbits sacrified at 28 and 33 days of age, respectively. The concentrations of cholic and glycoholic acids were shown to vary significantly with diet. The composition of the bile of young rabbits differed from that of adults.

Eastwood et al. (1976) ont montré que les acides biliaires (AB) étaient retenus par les constituants pariétaux des végétaux. L'acide désoxycholique (DOC) est celui dont le taux de fixation est le plus élevé comparativement aux autres acides; or c'est lui dont le taux est le plus élevé dans la bile du lapin et qui est présent tout au long du tractus digestif (Dehbi, 1984). Notre travail se propose, d'une part, d'étudier les variations qualitatives et quantitatives des $A B$ dans la bile et le tractus digestif du lapin en fonction de la nature du régime, d'autre part, d'étudier ces mêmes variations chez le lapereau au sevrage. Nous présentons ici les résultats qui ont trait à la bile vésiculaire des 2 catégories d'animaux.

Matériel et méthodes. Vingt-cinq lapins néozélandais mâles de $2,5 \mathrm{~kg}$ nourris ad libitum sont répartis en 3 groupes de 5,10 et 10 animaux. Ils reçoivent à volonté pendant 6 semaines les régimes suivants : $(T)$ aliment du commerce, $(P)$ aliment contenant $70 \%$ de pulpes de betteraves déshydratées, ( $L$ ) aliment contenant $70 \%$ de farine de luzerne déshydratée contenant respectivement 33, 41 et $42 \%$ de parois cellulaires (NDF \% de la matière sèche MS). La consommation journalière moyenne est de 163 (T), 132 (P) et 197 (L) g de M.S. Deux lots de 7 lapereaux sont sacrifiés après le sevrage, l'un à 28 jours (L1) et l'autre à 33 jours (L2) ; ce dernier lot reçoit l'aliment $T$ pendant 5 jours.

La bile est prélevée dans la vésicule biliaire après sacrification entre 15 et $18 \mathrm{~h}$. Les $A B$ libres et conjugués sont dosés par chromatographie en phase liquide : colonne de silice greffée $C 18,250 \mathrm{~mm}$ de long, diamètre $4 \mathrm{~mm}$, détection UV $210 \mathrm{~nm}$, phase mobile méthanol-eau 75-25 V/V (Armstrong et Carey, 1982). Les constituants pariétaux sont dosés par la méthode de Van Soest.

Résultats et discussion. Dans tous les cas l'acide dont le taux est le plus élevé est le HDC suivi du GDC (tabl. 1). L'HDC a été trouvé dans la bile, le plasma 
TABL. 1. - Concentration en acides biliaires de la bile vésiculaire en $\mu E q / \mathrm{m} /$ (moyenne du lot suivie de l'écart type de la moyenne).

\begin{tabular}{|c|c|c|c|c|c|c|}
\hline \multirow{2}{*}{ Acide } & \multicolumn{3}{|c|}{ Lapins } & \multicolumn{2}{|c|}{ Lapereaux } & \multirow{2}{*}{$\begin{array}{c}\text { Comparaison } \\
\text { de } \\
\text { moyennes }\end{array}$} \\
\hline & $\begin{array}{l}\text { Témoin } \\
\text { a (5) }\end{array}$ & $\begin{array}{c}\text { Pulpes }\left({ }^{*}\right) \\
\text { b }(8)\end{array}$ & $\begin{array}{l}\text { Luzerne } \\
\text { c (10) }\end{array}$ & $\begin{array}{l}28 \text { Jours } \\
d(7)\end{array}$ & $\begin{array}{l}33 \text { Jours } \\
\text { e (7) }\end{array}$ & \\
\hline $\begin{array}{l}\text { Glycocholique } \\
\text { (GC) }\end{array}$ & $\begin{array}{r}50 \\
(14)\end{array}$ & $\begin{array}{r}22 \\
(10)\end{array}$ & $\begin{array}{r}30 \\
(9)\end{array}$ & $\begin{array}{r}10 \\
(2)\end{array}$ & $\begin{array}{r}4 \\
(1)\end{array}$ & ad,ae, de : $x x$ \\
\hline $\begin{array}{l}\text { Glycochenodésoxycholique } \\
\text { (GCDC) }\end{array}$ & $\begin{array}{c}1,9 \\
(1,3)\end{array}$ & $\begin{array}{c}0,4 \\
(0,4)\end{array}$ & $\begin{array}{c}1,4 \\
(0,8)\end{array}$ & $\begin{array}{c}1,3 \\
(0,9)\end{array}$ & $\begin{array}{c}0,8 \\
(0,3)\end{array}$ & \\
\hline $\begin{array}{l}\text { Glycodésoxycholique } \\
\text { (GDC) }\end{array}$ & $\begin{array}{r}137 \\
(96)\end{array}$ & $\begin{array}{l}106 \\
(17)\end{array}$ & $\begin{array}{r}82 \\
(19)\end{array}$ & $\begin{array}{r}121 \\
(21)\end{array}$ & $\begin{array}{r}37 \\
(9)\end{array}$ & acetde $: x x$ \\
\hline $\begin{array}{l}6 \text { Cétolithocholique } \\
\quad(6 \mathrm{CLC})\end{array}$ & $\begin{array}{r}1 \\
(1)\end{array}$ & $\begin{array}{c}1,2 \\
(0,6)\end{array}$ & $\begin{array}{c}0,4 \\
(0,4)\end{array}$ & $\begin{array}{c}0,7 \\
(0,7)\end{array}$ & $\begin{array}{c}0,1 \\
(0,1)\end{array}$ & \\
\hline $\begin{array}{l}\text { Cholique } \\
\text { (C) }\end{array}$ & $\begin{array}{c}0,9 \\
(0,2)\end{array}$ & $\begin{array}{l}13,5 \\
(0,1)\end{array}$ & $\begin{array}{c}2,7 \\
(0,5)\end{array}$ & $\begin{array}{l}11,7 \\
(0,6)\end{array}$ & $\begin{array}{l}5 \\
(1,7)\end{array}$ & de $: x ; a e^{a b}, x x$,ad et \\
\hline $\begin{array}{l}\text { Hyodésoxycholique } \\
\text { (HDC) }\end{array}$ & $\begin{array}{l}168 \\
(30)\end{array}$ & $\begin{array}{l}162 \\
(13)\end{array}$ & $\begin{array}{r}278 \\
(48)\end{array}$ & $\begin{array}{l}225 \\
(15)\end{array}$ & $\begin{array}{l}248 \\
(9)\end{array}$ & $\begin{array}{l}\text { bc,ae, de : } x x \\
\text { ac : } x \mathbf{x x}\end{array}$ \\
\hline
\end{tabular}

$x, x x, x x x$ : Ecart significatif au seuil $P<0,01,0,05,0,10$. (*) Deux lapins de ce lot sont morts en cours d'expérience suite à une diarrhée.

et les contenus digestifs du lapin (Dehbi, 1984). Sacquet et al. (1983) ont montré qu'il pourrait être absorbé au niveau intestinal chez l'homme où on le caractérise dans la bile, le foie, l'urine et les fèces soit en gluco-soit en glucurono-conjugué. Les différences constatées entre les 2 régimes $L$ et $P$ ne sont significatives que pour le HDC dont la concentration est plus forte dans la bile des lapins recevant l'aliment L. La luzerne entraîne des concentrations plus fortes de HDC et de C alors que le régime témoin induit une teneur plus importante de GC et de GDC. Le taux du GDC varie en proportion inverse du taux de lignocellulose et de cellulose des 3 régimes.

Dans le cas des lapereaux les teneurs en $A B$ sont différentes de celles des lapins adultes. Des différences significatives apparaissent entre les 2 groupes L1 (sevrage) et L2 ( 5 jours après le sevrage) pour les taux d'HDC, de GDC, GC et C. Le sevrage entraîne au bout de 5 jours une diminution sensible du GC, du GDC et du $C$, et une augmentation du HDC.

En conclusion, il apparaît que la composition quantitative de la bile vésiculaire des lapereaux évolue avec le changement de leur alimentation. La teneur en fibres des régimes modifie la composition quantitative de la bile vésiculaire sans affecter la nature des $A B$ retrouvés.

Armstrong M. J., Carey M. C., 1982. J. Lipid Res., 23, 70-80.

Dehbi L., 1984. Thèse de $3^{e}$ cycle, Univ. Paris VI.

Eastwood M. A., Anderson R., Mitchell W. D., Robertson J., Pocooks S., 1976. J. Nutr., 106. $1429-1432$.

Sacquet E., Parquet M., Riottot M., Raizman A., Jarrige P., Huguet C., Infante R., 1983. J. LipidRes. 24, 604-613. 